\title{
A Noninvasive Transcutaneous Alternative to Rectal Thermometry for Continuous Measurement of Core Temperature in the Piglet
}

\author{
SHAUL DOLLBERG, YURUI XI, AND MICHAEL M. DONNELLY
}

Department of Pediatrics, Division of Neonatology, University of Cincinnati College of Medicine and Children's Hospital Medical Center, and the Perinatal Research Institute. Cincinnati, Ohio 45267

\begin{abstract}
Deep body temperature is an important and accepted index of health status in newborn infants. There are no easily used accurate methods for continuous deep body temperature measurements. Oral and tympanic membrane temperatures correlate well with rectal temperature but are not easily adapted to continuous measurement. We devised a noninvasive transcutaneous temperature sensor for continuous deep body temperature measurement that relies on the principle that, under steady state conditions, the temperature at the thermally insulated surface of a warm body, i.e. a zero heat loss surface, will be in equilibrium with the warmest part of the body. We used a standard clinical temperature probe placed between the skin and the mattress and attached to the skin with a foam adhesive disk. We used standard skin temperature probe attachment disks, which are also designed to provide thermal insulation to the skin temperature probe. We tested the hypothesis that this transcutaneous temperature would track body temperature as indicated by rectal temperature. In six anesthetized (pentobarbital) newborn piglets $(1600 \pm 200$ g) placed on their abdomen in a convectively warmed infant incubator, we measured continuously the following temperatures for $5 \mathrm{~h}$ : transcutaneous over lower and upper abdomen, brown fat, rectal, and descending aorta. To examine the influence of environment, we varied the incubator air temperature between $32^{\circ}$ and $36^{\circ} \mathrm{C}$. Both transcutaneous temperatures tracked the rectal temperature, within $0.2^{\circ} \mathrm{C}$ for transcutaneous over lower abdomen and within $0.3^{\circ} \mathrm{C}$ for transcutaneous over upper abdomen. Aortic artery temperature was always lower than rectal. We conclude that transcutaneous temperature has the potential to be used reliably as a noninvasive replacement for continuous rectal temperature monitoring. (Pediatr Res 34: 512-517, 1993)
\end{abstract}

Vital signs monitoring of preterm infants in the neonatal intensive care unit, until recently, has been predominantly invasive. This reliance on invasive measurement systems stems from the difficulty in obtaining reliable physiologic or biochemical measurements through indirect methodologies, for example $\mathrm{PO}_{2}$ in the blood through the skin as in transcutaneous $\mathrm{PO}_{2}(1)$. The development of noninvasive transcutaneous transduction systems for blood gases, heart rate, respiratory rate, and blood pressure have made continuous monitoring of these vital parameters commonplace in the care of the preterm infant. However,

Received February 15, 1993; accepted May 26, 1993

Correspondence and reprint requests: Michael M. Donnelly, Dept. of Pediatrics. College of Medicine. University of Cincinnati, 231 Bethesda Ave., Cincinnati, OH 45267-0541.

Supported in part by NIH HD 20761-06 (M.M.D.). Project 5 of the Perinatal Emphasis Research Center. the determination of core temperature, a widely used index for health status, remains predominantly an invasive measurement (2). Moreover, continuous monitoring of this vital parameter is not a routine clinical assessment because of the difficulty of implementing a safe and a convenient noninvasive measurement system. The most reliable and widely used measurement site for core temperature continues to be the rectum: measurement of rectal temperature is highly invasive and not suitable for continuous use in the nonanesthetized patient (3). In addition, the depth of insertion is crucial for obtaining accurate and reproducible measurements (2).

A transcutaneous temperature measurement system that could provide repeatable deep body temperature measurements could be a valuable monitor of health status in critically ill infants as well as in adults. We developed a simple transcutaneous temperature measurement system that uses a highly insulated temperature transducer to attain temperature equilibrium between the device and the warm core. The concept for our device was based on the established principle of "zero heat flow" $(4,5)$. We designed our studies to test the hypothesis that the transcutaneous temperature from our simple device would match core temperature as indicated by rectal temperature. Infants cared for in incubators, radiant warmers, or open bassinets always have a significant portion of their surface in contact with the mattress of the particular device. These mattresses provide a high degree of insulation with resultant very low heat loss by conduction from the surface of the infant to the mattress (6-9). Under most circumstances in an incubator, the exposed skin will be at a lower temperature than that in contact with the mattress because of the insulation provided by the mattress itself. This common condition where a significant part of the surface of infants is usually thermally insulated by the bassinet, incubator, or radiant warmer mattress creates an ideal situation to promote a zone of zero heat flow, using a relatively small thermal insulator. We have designed a device that relies on the supplemental thermal insulation provided by infant mattresses to achieve a condition of zero heat flow, with a response time acceptable for most clinical situations. We tested the hypothesis that the transcutaneous temperature probe will track core temperature as indicated by simultaneous rectal temperature recordings.

\section{MATERIALS AND METHODS}

Zero heat flow temperature measurement principle. The transcutaneous temperature measurement principle is described in Figures 1 and 2. There is an engineering technique commonly used to ascertain the temperature of objects whose interiors are warmer than their exteriors $(4,5,10,11)$. This technique is referred to as the "zero heat flow principle." The principle relies on the fact that any body that has an internal heat-producing component will have a continuous flow of heat to its surface as long as the surface is cooler than its internal heat-producing 

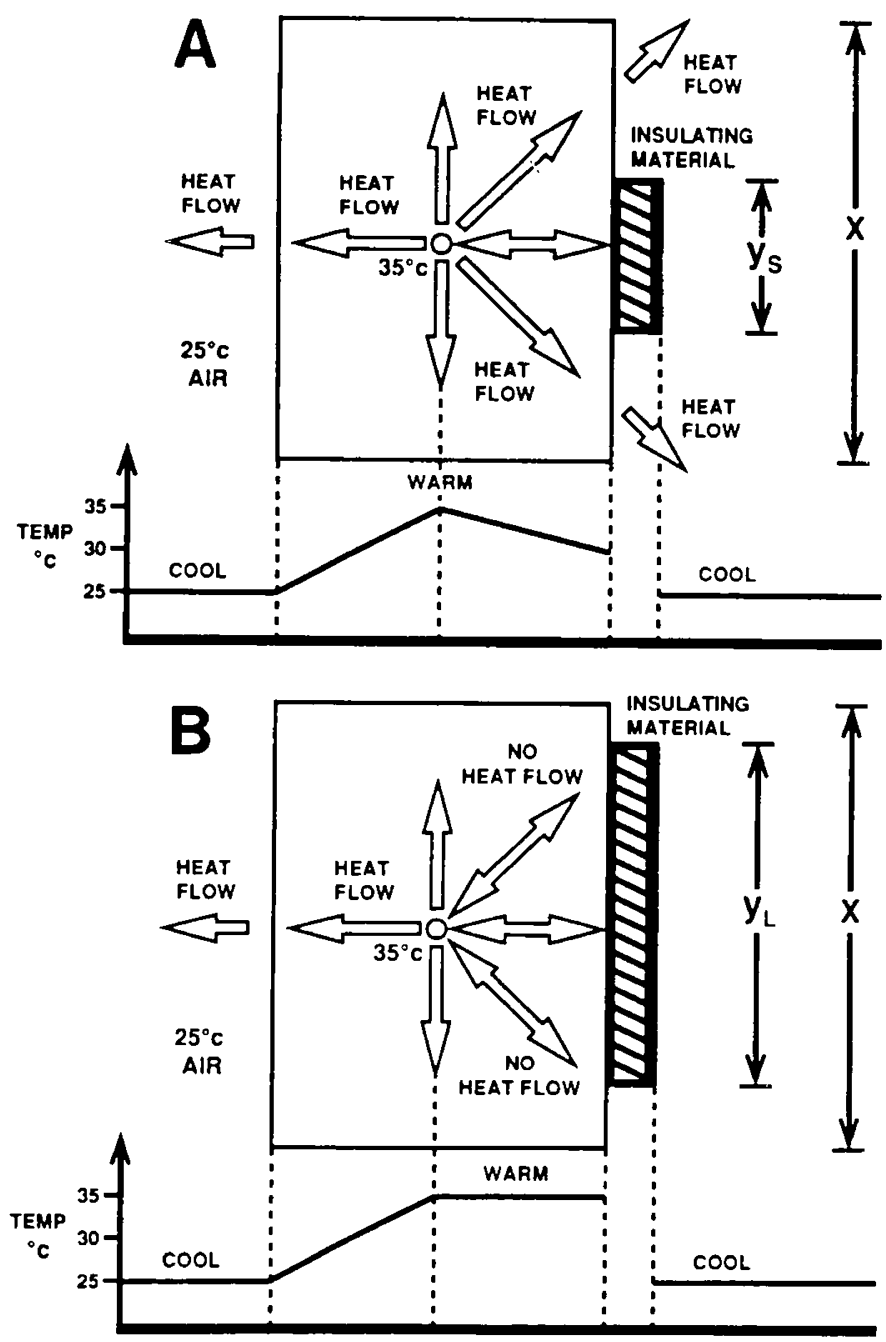

Fig. 1. A, A body as shown with a heat-producing core at $35^{\circ} \mathrm{C}$ will have a continuous heat flow to its surface, which is exposed to an air temperature of $25^{\circ} \mathrm{C}$. Heat continues to flow as long as this gradient is maintained. When one side (length $x$ ) of the body is partially insulated by an insulating material (length $y_{s}$ ), there will be an interruption in heat loss from this insulated surface. Heat will continue to flow to the uninsulated part of the surface (length $x-y_{s}$ ), but the gradient structure between the core and the insulated surface will be smaller than that of the uninsulated surface. $B$, When the length of the insulating material as shown in $A$ is increased to length $y_{L}$. there will be a further decrease in the amount of heat loss from the insulated surface. By increasing the size of the insulation, it is possible to block the flow of heat and decrease the gradient between the core and the surface such that the temperature at this insulated surface is the same as the core of the body.

component (12-14). The amount of heat flowing to the surface will be a function of the temperature gradient between the surface and the heat-producing component, and the overall thermal conductivity of the body. Large temperature gradients between the warm center and cooler surfaces will produce higher heat flow to the surface than smaller gradients. As shown in Figure $1 A$, there is a temperature gradient from the warmest component of the body to the surface. If one side of the body is perfectly insulated, as shown in Figure $1 B$, heat will continue to flow to this surface and, because this heat cannot be dissipated, there will be a gradual increase in the temperature of this insulated surface. The temperature of the surface will continue to increase and the amount of heat flowing to this surface will continue to decrease because the temperature gradient is getting smaller. Eventually, the temperature of the surface will reach the temperature of the warmest part of the body and heat will stop flowing

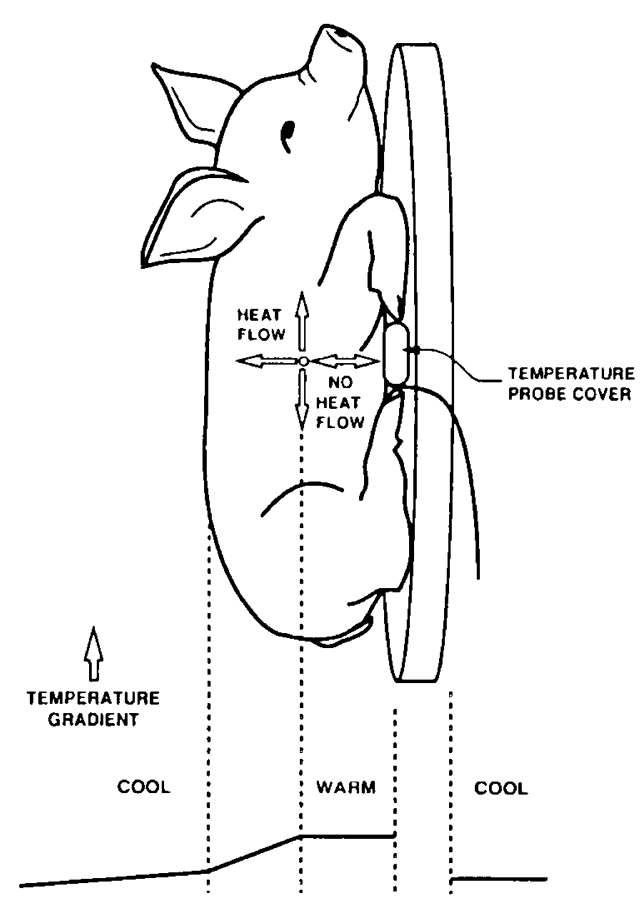

Fig. 2. As shown, we attached the temperature probe to the abdomen of the pig between the skin and the mattress of the incubator. The temperature probe cover and the mattress provide a degree of insulation that prevents heat flow from this surface.

to the surface. When this temperature equilibrium is achieved, a temperature probe located between the surface and the thermal insulator will register the temperature of the warmest part of the body. The time to reach this point of temperature equilibrium will be dependent on the thermal conductivity of the body and the area of the insulated surface relative to the size of the body. This theory of solid body conductivity was applied to our piglet model. It should be mentioned, however, that mammalian tissues may transfer heat through the circulation in addition to conduction; this form of heat transfer is referred to as convective flow, but it is merely another form of conduction. Thus, it is important to recognize that heat flow to the surface and therefore the time response of the system may be different in extremes of clinical conditions such as hyperthermia with vasodilatation or hypothermia with vasoconstriction.

A large body with a very small insulated surface area may never reach temperature equilibrium to achieve no heat flow, because there will be a continuous flow of heat to the cooler surfaces and the environment, as shown in Figure $1 A$. Increasing the surface area of the insulator as shown in Figure $1 B$ will result in a decrease in the temperature gradient between the warm core and the insulated surface compared with the temperature gradient of a smaller insulated area. Using this technique, it is possible to develop geometries for an insulated temperature probe that achieves a negligible temperature gradient between the warm core and the insulated surface which suits the mechanical and thermal dimensions of any body which has an internal heat producing component (15).

The rate of heat flow depends on the temperature gradient across the body and the thermal conductance of the object. In practice in existing incubators and radiant warmers. heat loss by conduction is virtually eliminated. When a material with a very low thermal conductivity is used, it is possible to reduce the conductive heat loss to near 0 . Foam mattresses used in infant incubators and radiant warmers have the thermal conductivity as low as $0.02 \mathrm{~W} / \mathrm{m}^{\circ} \mathrm{C}$.

Piglet preparation. Six newborn piglets, $1600 \pm 200 \mathrm{~g}$ (mean $\pm \mathrm{SD}$; range $1350-1800 \mathrm{~g}), 3$ to $6 \mathrm{~d}$ old, were anesthetized with pentobarbital and intubated to maintain a patent airway. The 
piglets were maintained thermally stable using a servo-controlled heat pad (Electric Pad Service Inc., Hattisburg, MI). Micro YSI temperature probes (YSI model 521, YSI Inc., Yellow Springs, $\mathrm{OH})$ were used to measure arterial, rectal, lower abdomen, upper abdomen, and midscapular (just above the brown adipose tissue region) temperatures. A micro needle temperature probe (YSI model 511, YSI Inc.) was used to measure brown adipose tissue temperature. After the anesthesia, we exposed the femoral artery and inserted a YSI micro temperature probe, ensuring that the tip of the probe was at the descending aorta. Another temperature probe was inserted $7 \mathrm{~cm}$ into the rectum. We attached a probe to the lower abdomen of the piglet, directly below the umbilicus, using a disk insulator (part 203-1980-300, Ohmeda, Columbia, MD). This insulator is routinely used to attach skin temperature probes to the skin of preterm infants cared for in incubators or radiant warmers. Another probe was attached with the same type of disk insulator to the upper abdomen just below the xiphoid process. The micro needle temperature probe was inserted into the brown fat, which was identified transdermally by a handheld infrared thermometer (model C600M, Biotherm Infrared Thermometer, Linear Laboratories, Sunnyvale, CA). The ipsilateral femoral artery was exposed and catheterized for blood pressure measurement, and a femoral vein was catheterized and served as venous access for further anesthesia. To continuously assess the health status of the pig, we measured continuous blood pressure, using the arterial catheter with a pressure transducer connected to a Gould pressure amplifier and strip chart recorder. In addition, we performed hourly arterial blood gas and glucose determinations.

Protocol design. We then placed the piglets in an infant incubator (model C100, Air Shields-Vickers Inc., Hatboro, PA) that was prewarmed to $32^{\circ} \mathrm{C}$ air temperature. We allowed a $45-\mathrm{min}$ equilibration period for the piglet to adjust to the incubator environment before we commenced recording temperatures. We used a computerized data logging system (Asystant Plus running on an IBM model PS 2/286, Asyst Software Technologies Inc., Rochester, NY) to record all temperatures. The computer was programmed to convert the indicated temperatures from the YSI probes to actual temperatures using the temperature calibration data for each individual probe (calibration curves certified to National Institute of Standards and Technology). The data acquisition rate was one sample every $5 \mathrm{~s}$ for all measured variables.

The following experimental protocol was used for each piglet. During the first hour, the piglet was prepared for the experiment while lying on the heated pad in room air. It was then placed in the incubator, which was prewarmed to $32^{\circ} \mathrm{C}$ air temperature. Temperature data collection was started after $45 \mathrm{~min}$ of equilibration in the prewarmed incubator. To examine the influence of a change in environmental temperature on the response of the transcutaneous temperature measurement system, we increased the incubator air temperature set point to $36^{\circ} \mathrm{C}$ after achieving steady state at $32^{\circ} \mathrm{C}$ air temperature. Forty-five min after reaching steady state at an air temperature of $36^{\circ} \mathrm{C}$, we returned the air temperature set point to $32^{\circ} \mathrm{C}$. All temperatures were recorded for at least $3 \mathrm{~h}$ for each piglet.

\section{RESULTS}

The difference or gradient between rectal temperature and the insulated skin temperature recordings of the lower and upper abdomen, and between rectal and arterial temperature, was computed for each set of measurements, which were made every $5 \mathrm{~s}$. The means and SD of these data for each piglet are summarized in Table 1. We then used these gradients from the six piglets studied to calculate the means and associated SD for each temperature gradient for the group. The insulated temperature probe that was placed between the lower abdomen and the mattress gave the best correlation with rectal temperature. The range of temperature difference between rectal and lower abdomen transcutaneous temperature was $+0.2^{\circ}$ to $-0.1^{\circ} \mathrm{C}$. The mean
Table 1. Difference or gradient between rectal temperature and insulated skin temperature recordings of lower and upper abdomen and between rectal and arterial temperature*

\begin{tabular}{crrr}
\hline $\begin{array}{c}\text { Pig } \\
\text { no. }\end{array}$ & $\begin{array}{c}\text { Rectum-upper } \\
\text { abdomen }\end{array}$ & \multicolumn{1}{c}{$\begin{array}{c}\text { Rectum-lower } \\
\text { abdomen }\end{array}$} & \multicolumn{1}{c}{ Rectal-arterial } \\
\hline 1 & $1.133 \pm 0.175$ & $-0.049 \pm 0.106$ & $0.396 \pm 0.585$ \\
2 & $0.102 \pm 0.054$ & $-0.033 \pm 0.056$ & $0.356 \pm 0.025$ \\
3 & $-0.096 \pm 0.090$ & $-0.011 \pm 0.077$ & $0.330 \pm 0.030$ \\
4 & $-0.105 \pm 0.509$ & $-0.044 \pm 0.052$ & $0.279 \pm 0.475$ \\
5 & $0.106 \pm 0.097$ & $0.106 \pm 0.064$ & $0.699 \pm 0.095$ \\
6 & $0.113 \pm 0.046$ & $-0.044 \pm 0.068$ & $-0.290 \pm 0.074$ \\
\hline
\end{tabular}

* Differences were computed for each set of measurements. which were made every $5 \mathrm{~s}$. Values are means \pm SD.

Table 2. Differences of upper abdomen, lower abdomen, and arterial temperatures from rectal temperature in six piglets during entire study period*

\begin{tabular}{lrrr}
\hline $\begin{array}{c}\text { Air } \\
\text { temperature }\end{array}$ & $\begin{array}{c}\text { Rectal-lower } \\
\text { abdomen }\end{array}$ & $\begin{array}{c}\text { Rectal-upper } \\
\text { abdomen }\end{array}$ & Rectal-arterial \\
\hline $32^{\circ} \mathrm{C}$ & $0.00 \pm 0.02$ & $0.05 \pm 0.02$ & $0.37 \pm 0.04$ \\
$32^{\circ}->36^{\circ} \mathrm{C}$ & $-0.02 \pm 0.02$ & $-0.08 \pm 0.04$ & $0.45 \pm 0.04$ \\
\hline
\end{tabular}

* Values are means $\pm \mathrm{SD}$.

and SD of the differences of each monitored temperature site from rectal temperature for the six piglets are summarized in Table 2. When the air temperature in the incubator was changed to $36^{\circ} \mathrm{C}$, there was a concomitant increase in rectal temperature.

A representative set of the data obtained every $5 \mathrm{~s}$ from one piglet is shown in Figure $3 A$. This figure represents data obtained over $40 \mathrm{~min}$, during the period when the air temperature was increased from $32^{\circ}$ to $36^{\circ} \mathrm{C}$. The temperatures at each site exhibited a tracking behavior, showing concomitant increases and decreases with rectal temperature. There was a consistent sitedependent offset between rectal and each of the other temperatures. The lower abdomen skin temperature follows all changes in rectal temperature with a minimal lag period. Even during the period when the air temperature of the incubator was increased and decreased from a steady state condition, the skin temperature of the lower abdomen tracked rectal temperature within the range of $+0.3^{\circ}$ to $-0.1^{\circ} \mathrm{C}$. Arterial temperature, measured in the descending aorta, was always lower than rectal temperature in each of the six piglets studied.

A difference of $0.37 \pm 0.04^{\circ} \mathrm{C}$ between the arterial and rectal temperatures was observed when the air temperature was maintained at $32^{\circ} \mathrm{C}$ (mean $\pm \mathrm{SEM}$ ) (Fig. $3 B$ ). This difference, with the rectal temperature always higher than arterial temperature, was present also when the piglet was studied in room temperature or when the air temperature was elevated to $36^{\circ} \mathrm{C}$. Manipulating the piglet inside the incubator (i.e. reversing the position of head or tail with respect to the air inlet in the incubator, or moving the animal on the mattress) did not change the temperature gradients between the skin abdomen and rectal probes. The temperature of the brown fat, measured by both the transcutaneous and the intratissue probes, was always lower than rectal temperature. This indicates that brown fat thermogenesis was not active and confirms that for the environmental temperature range of $32^{\circ}$ to $36^{\circ} \mathrm{C}$ used in our studies the piglets were not cold stressed.

\section{DISCUSSION}

Our study shows that insulated transcutaneous temperature data match core temperature as indicated by rectal temperature within $0.2^{\circ} \mathrm{C}$. The rectum, axilla, and tympanic membrane are the predominant sites used clinically for measurement of deep body temperature in the newborn infant. Rectal temperature continues to be recognized as the "gold standard" for core temperature measurement in clinical practice (16). This is be- 

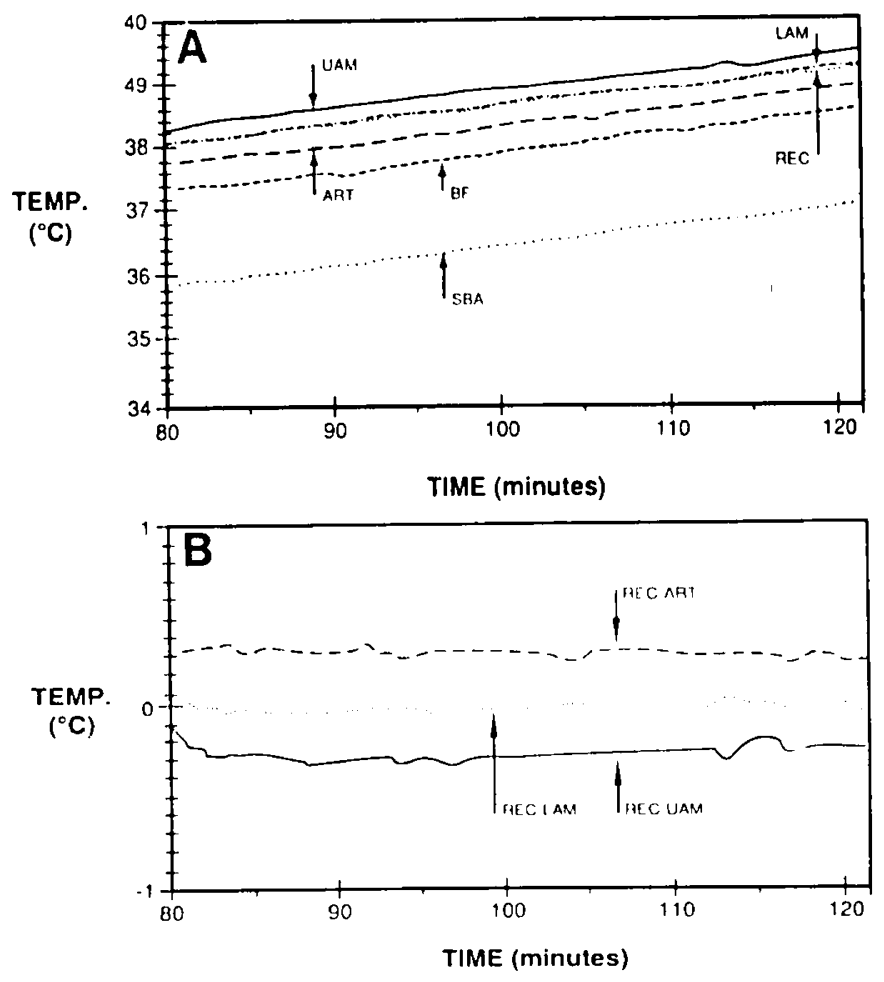

Fig. 3. A. A representative sample of the actual temperature data obtained from that part of the experiment immediately after increasing the temperature from $32^{\circ}$ to $36^{\circ} \mathrm{C} . B F$, brown fat temperature: $S B .4$, skin over brown fat temperature: $R E C$, rectal temperature: $A R T$, arterial temperature; $L A M$ and $U A M$, temperatures recorded by the thermally insulated probes between the lower and upper abdomen. respectively, and the mattress. All temperatures had concomitant increases with rectal temperature as the air temperature in the incubator was increased from $32^{\circ}$ to $36^{\circ} \mathrm{C} . B$. The differences between rectal $\left(R E C^{\circ}\right)$ and arterial $(A R T)$. lower abdomen $(L \cdot M)$ and upper abdomen (U.AM) temperatures are shown. These data are from the same study period as shown in $A$. The gradients were obtained by subtracting the various temperatures from rectal temperature. The REC-LAM gradient was $<0.1^{\circ} \mathrm{C}$ and the RECART and REC-UAM gradients were $<0.3^{\circ} \mathrm{C}$.

cause of the accessibility of the rectum, a deep site suitable for continuous clinical assessment of fever states, and because rectal temperature is always higher than that measured at any other site in the body. Studies in human subjects showed that mean rectal temperature is $0.35 \pm 0.01^{\circ} \mathrm{C}$ higher than oral temperature (17), $0.22 \pm 0.15^{\circ} \mathrm{C}$ higher than right ventricular blood temperature (18), and $0.23 \pm 0.14^{\circ} \mathrm{C}$ higher than pulmonary arterial temperature (19). The possibility of the effect of bacterial heat production has been studied by sterilization of the colon. It was found that the apparent bacterial effect is insignificant $(20,21)$. The reason for rectal temperature measurement being higher than that of any other body organ is not known. Rectal thermometry, although suitable for intermittent and continuous temperature measurement in adults, is not recommended or used routinely in neonates and small children because of the rare but distinct possibility of perforation (22). Under non-steady state, rectal temperature in adults was shown to lag temperatures from other sites used to measure deep body temperature (23).

In infants, the axilla is a convenient and commonly used site for intermittent and continuous measurement of core temperature. Standard skin-based temperature probes can be easily attached for long-term continuous measurement and observation at this skin site. It has been shown that for a mercury-in-glass thermometer the temperature should be read $10 \mathrm{~min}$ after placing the thermometer in the axilla (2). Axillary temperatures are consistently lower than oral and deep rectal temperatures, but the gradient decreases as the time of measurement increases from 10 to $20 \mathrm{~min}(24,25)$. In measuring axillary temperature, the arm serves an insulating function for the temperature probe and helps to shield it from the influence of the environment. Because the perfusion of tissues in the arm and the axilla is relatively high, the measured temperature of an insulated temperature probe at this site will be highly influenced by environmental conditions, in particular air temperature, air velocity, and relative humidity. This high rate of perfusion promotes high thermal conduction to the surface with concomitant heat loss from these surfaces; so at these sites, significantly greater levels of insulation will be required to decrease the temperature gradient between the surface and the deeply perfused tissue. These sites are therefore unsuitable for the noninvasive measurement of deep body temperature without the incorporation of large insulating surfaces.

Tympanic membrane thermometry has been widely used and studied. The temperature of the tympanic membrane has been shown to closely approximate that of the arterial temperature (26). The temperature of the tympanic membrane is thought to be close to arterial temperature because the blood vessels surrounding the tympanic membrane branch from the main artery, which delivers blood to the hypothalamus, which is recognized as the predominant site for the control of body temperature. Tympanic thermometry has been based on measurements of the membrane temperature by direct contact with temperature thermistor or thermocouple and has been found to be a reliable index of core temperature (2). However, this method causes the patient discomfort and necessitates skill in the insertion and attachment of the temperature sensor. Perforation of the tympanic membrane has been reported (27) and is a definite risk with the use of this site for contact temperature measurement. Infrared tympanic thermometry is a new technique that allows rapid measurement of tympanic membrane temperature, with the advantage of minimal intrusion and complication, and requires minimal personnel training. Studies in adults and infants have shown the infrared tympanic thermometer to be accurate between $34.0^{\circ}$ and $39.5^{\circ} \mathrm{C}$, with deviations from pulmonary artery temperatures of $-0.2^{\circ}$ to $-0.4^{\circ} \mathrm{C}(26)$. Freed and Fraley (28) recently reported on the lack of agreement of tympanic temperature assessments with conventional methods. However, current technology does not allow for tympanic thermometry to be conducted on a continuous basis, and the infrared tympanic devices are still too large for the auricular cavity of preterm infants.

A consistent effort to decrease the invasiveness of medical monitoring, to decrease trauma and risks of infection. has led to transcutaneous measurements of mechanical forces (noninvasive blood pressure), cardiac and brain electrical activity (ECG and EEG), evoked potentials, and the presence of clinically important biochemical parameters such as oxygen, carbon dioxide, and oxygenated $\mathrm{Hb}\left(\mathrm{PO}_{2}, \mathrm{PCO}_{2}\right.$, and $\mathrm{O}_{2}$ saturation $)$.

The zero heat flow principle has been used primarily for continuous measurement of temperatures in hostile industrial environments, for example to measure temperatures inside hot steam pipes by attaching a temperature probe to the surface of the pipe. The time constant for the application of this principle will vary according to the thermal conductivity of the specific material in each application. The time to reach temperature equilibrium will be short for objects that have a high thermal conductivity. In human applications, the thermal conductivity of perfused skin is high (estimated at $200-300 \mathrm{~W} /\left(\mathrm{cm} \cdot{ }^{\circ} \mathrm{C}\right)(17$. 29,30 ) and approaches the thermal conductivity of copper [ 250 $\left.350 \mathrm{~W} /\left(\mathrm{cm} \cdot{ }^{\circ} \mathrm{C}\right)\right]$. Also, the mattress provides near perfect insulation (thermal conductance $<0.02 \mathrm{~W} / \mathrm{m}^{\circ} \mathrm{C}$ ) and complements the insulation properties of the foam attachment disk.

There is one skin-mattress temperature measurement study in newborn infants by Nasim-Khan et al. (31). This study examined the differences between rectal temperature and the temperature 
between the skin and the mattress using large mercury-in-glass thermometers. These investigators measured a difference between rectal and transcutaneous temperature of $0.58 \pm 0.19^{\circ} \mathrm{C}$ (mean $\pm \mathrm{SEM}$ ) for term infants and $0.26 \pm 0.19^{\circ} \mathrm{C}$ for preterm infants. The mercury-in-glass thermometer has a long time constant and a large contact surface, which makes it highly influenced by environmental artifact unless properly insulated. This could explain why in this study, in addition to the high degree of deviation between rectal and skin-mattress temperatures, there was not a concomitant change in the temperature as measured by the skin-mattress thermometer when the temperature as measured rectally showed significant changes.

A thermometer using the zero heat flow principle, was proposed by Fox et al. (5). The probe they used was heated to $37^{\circ} \mathrm{C}$, thus creating a theoretical zone of zero heat flow from the core to the surface. The results of their preliminary study were encouraging, but did show offsets of up to about $0.5^{\circ} \mathrm{C}$ under conditions of endogenous changes in body temperature. This zero heat flow principle probe was commercially produced after the publication of the study results; however, the equilibration time for the commercial probe was up to $20 \mathrm{~min}$ because of the time for the active sensing element to reach a temperature of $37^{\circ} \mathrm{C}(32)$. No further studies have been reported to investigate the influence of changes in body temperature on the accuracy or time response of this technique. We speculate that any change in body temperature from $37^{\circ} \mathrm{C}$ will result in a heat flow gradient between the core and the preheated probe, which was designed to have a fixed temperature of $37^{\circ} \mathrm{C}$. Zero heat flow will be eliminated when body temperature deviates from $37^{\circ} \mathrm{C}$, thus invalidating the measurement principle.

In a study by LaMont-Gregory et al. (33; personal communication), in which the authors used a special material to provide thermal insulation of the probe, a difference of $0.01 \pm 0.07^{\circ} \mathrm{C}$ (mean $\pm \mathrm{SD}$ ) between skin-mattress and rectal temperatures in 90 normal newborns was found. This study was conducted on thermally stable sleeping infants. The range of differences between insulated skin temperature and rectal temperature reported in their study was $\pm 0.2^{\circ} \mathrm{C}$. These data confirm the viability of the basic principle of zero heat flow in thermally stable infants in a stable environment. This study did not examine the influence of endogenous or exogenous temperature changes on the accuracy or response time of the transcutaneous technique.

Continuous temperature measurement using the zero heat flow principle has not previously been studied in an animal model. Additionally, the accuracy and response times for this technique have not been previously characterized under changing environmental conditions. We have demonstrated that the physical principle of zero heat flow is valid in living perfused tissues and that transcutaneous skin-mattress temperature tracks rectal temperature across a wide range of environmental temperature and even under conditions of changing body temperature. The response time of our skin-mattress temperature system is short (with a time constant estimate in our studies of less than $35 \mathrm{~s}$ ). This small time constant results in minimal lag between changes in rectal temperature and that detected by the skin abdomen temperature, thereby making it applicable to clinical measurements of deep body temperature.

Applying this transcutaneous temperature measurement technique could significantly reduce the time required for routine body temperature measurement in the full-term nursery because the temperature probe could be left in place between measurements. This could completely eliminate all response time issues. This noninvasive transcutaneous temperature measurement technique could easily be applied to provide continuous measurement of deep body temperature in neonatal patients thermally supported in closed convective incubators or open radiant warmers. The availability of continuous deep body temperature with this population of infants could provide new insight into their thermal and metabolic status. The newborn piglet model, although close in weight to some preterm infants, is more mature at birth, having a high degree of insulation for heat loss and a lower overall flow of heat to the surface of the skin because of its low conductivity. These properties make the piglet potentially less responsive than even a full-term infant of similar weight would be. Care would need to be exercised to ensure that this transcutaneous temperature is not used for servocontrol of infant temperature until further research is completed and the relationship of this new temperature to the commonly used infant temperature sites has been developed.

\section{REFERENCES}

1. Stevenson DA. Benitz WE, Ariano RL. Stevenson DK 1992 Noninvasive methods for estimating in vivo oxygenation. Clin Pediatr 27:258-273

2. Togawa T 1985 Temperature measurement. Clin Phys Physiol Meas 6:83-108

3. Vale RJ 1981 Monitoring of temperature during anesthesia. Int Anesthesiol Clin 19:61-83

4. Fox RH, Solman AJ 1971 A new technique for monitoring the deep body temperature in man from the intact skin surface. J Physiol 212:8P-10P

5. Fox RH. Solman AJ. Isaacs R. Fry AJ. MacDonald IC 1973 A new method for monitoring deep body temperature from the skin surface. Clin Sci 44:8186

6. Bell EF, Gray C. Weinstein MR, Oh W 1980 The effects of thermal environment on heat balance and insensible water loss in low birthweight infants. $J$ Pediatr 96:452-459

7. Baumgan S 1985 Partitioning of heat losses and gains in premature newborn infants under radiant warmers. Pediatrics 75:89-99

8. Wheldon AC, Rutter $\mathrm{N} 1982$ The heat balance of small babies nursed in incubators and under radiant warmers. Early Hum Dev 6:131-143

9. Hey EN, Mount LE 1967 Heat losses from babies in incubators. Arch Dis Child 42:75-84

10. Singer B. Lipton B 1975 Monitoring of core temperature through the skin: a comparison with esophageal and tympanic temperatures. Bull NY Acad Med 51:947-952

11. Ball SG, Chalmers McM. Morgan AG, Solman AJ, Losowsky MS 1973 A clinical appraisal of transcutaneous deep body temperature. Bio Med 18:290 294

12. Breuer H 1924 The thermal conductivity of muscle and fat. Pfluegers Arch Gesamte Physiol Menschen Tiere 204:442-447

13. Spells KE 1960 The thermal conductivities of some biological fluids. Phys Med Biol 5:139-153

14. Stoll AM. Chianta MA. Piergallini JR 1979 Thermal conduction effects in human skin. Aviat Space Environ Med 50:778-787

15. LeBlanc M. Donnelly MM 1993 Thermoregulation, the design of incubators and radiant warmers. In: Braun Y (ed) Perinatal Biotechnology. Cambridge University Press, Cambridge. UK (in press)

16. Weiss ME, Reagan MS. France N 1991 Axillary vs. rectal temperature in ambulatory and hospitalized children. Pediatr Inf Dis J 10:541-542

17. Cranston WI. Gerbrandy J. Snell ES 1954 Oral, rectal and esophageal temperatures and some factors affecting them in man. J Physiol (Lond) 126:347358

18. Eichina LW, Berger AR. Rader B. Becker WH 195 I Comparison of intracardiac and intravascular temperatures with rectal temperatures in man. J Clin Invest 30:353-359

19. Ilsley AH, Rutten AJ. Runciman WB 1983 An evaluation of body temperature measurement. Anaesth Intensive Care 11:31-39

20. Rubin A. Horvath SM, Mellette HC 195I Effect of fecal bacterial activity on rectal temperature of man. Proc Soc Exp Biol Med 76:410-411

21. Duroyote AO, Garyson J 1971 Heat production in the gastrointestinal tract of the dog. J Physiol (Lond) 214:417-426

22. Vale RJ 1981 Monitoring of temperature during anesthesia. Int Anesthesiol Clin 19:61-83

23. Cooper KE, Kenyon JR 1957 A comparison of temperature measured in the rectum. esophagus and on the surface of the aorta during hypothermia in man. Br J Surg 44:616-619

24. Machino R 1959 Studies on clinical thermometry. J Jpn Assoc Phys Med Balneol Climatol 22:292-318

25. Mayfield SR. Bhatia J, Nakamura KT, Rios GR, Bell EF 1984 Temperature measured in term and preterm neonates. J Pediatr 104:271-275

26. Shinozaki T, Deane R. Perkins FM 1988 Infrared tympanic thermometer: evaluation of a new clinical thermometer. Crit Care Med 16:148-150

27. Tabor MW. Blaho DM, Schriver WR 1981 Tympanic membrane perforation: complication of tympanic thermometry during general anesthesia. Oral Surg 51:581-583

28. Freed GL. Fraley JK 1992 Lack of agreement of tympanic membrane temperature assessments with conventional methods in a private practice setup. Pediatr Inf Dis J 89:384-386 
29. Sulyok E. Jequier E. Prod'hom LS 1973 Thermal balance of the newborn infant in a heat-gaining environment. Pediatr Res 7:888-900

30. Chato JC 1987 Thermal properties of tissues. In: Skalak R. Chien S (eds) Handbook of Bioengineering. McGraw Hill, New York

31. Nasim-Khan M. Ahmad SH, Fakir S 1990 Comparing of temperatures at different sites in term and preterm neonates. Indian Pediatr 27:807-809

32. Singer B, Lipton B 1975 Monitoring of core temperature through the skin: a comparison with esophageal and tympanic temperatures. Bull NY Acad Sci 51:947-952

33. LaMont-Gregory E, Koppe JG. Lesnik Oberstein SY. Rvan TJ 1990 A minimally intrusive method for transcutaneous measurement of deep body temperature in infants. XII European Congress of Perinatal Medicine. Lyon. France, September 11-14

\section{Announcement}

\section{Society for Adolescent Medicine Annual Meeting}

The Society for Adolescent Medicine, a multidisciplinary professional organization. will hold its annual meeting in Los Angeles. CA, between March 16,1994 and March 20,1994 at the Century Plaza Hotel. The theme for the meeting will be "Adolescents and the Media." In addition to addressing this topic, the meeting will present new material on a broad range of issues important to adolescent physical and emotional health, including AIDS and HIV medical management, teenage sexuality, eating disorders, depression, and risk-taking behaviors, which encompass drug and alcohol use and abuse. Meeting presentations include all-day institutes, 3-hour clinically oriented workshops, luncheon seminars, scientific research paper presentations and poster sessions, as well as the prestigious Gallagher Lecture Series. CME/CEUs are available. For further information, contact the Society for Adolescent Medicine, Suite 120, 19401 E. 40 Highway, Independence, MO 64055. (816) 795-8336. 